\title{
Current treatment strategies for Philadelphia chromosome-positive adult acute lymphoblastic leukemia
}

\author{
Han-Seung Park \\ Department of Hematology, Asan Medical Center, University of Ulsan College of Medicine, Seoul, Korea
}

p-ISSN 2287-979X / e-ISSN 2288-0011 https://doi.org/10.5045/br.2020.S006 Blood Res 2020;55:S32-S36.

Received on December 13, 2019 Accepted on January 20, 2020

\author{
Correspondence to \\ Han-Seung Park, M.D. \\ Department of Hematology, Asan Medical \\ Center, University of Ulsan College of \\ Medicine, 88 Olympic-ro 43-gil, \\ Songpa-gu, Seoul 05505, Korea \\ E-mail: ennuyes@gmail.com \\ (C) 2020 Korean Society of Hematology
}

\begin{abstract}
Acute lymphoblastic leukemia (ALL) is an aggressive hematological disease. The incorporation of tyrosine kinase inhibitors (TKIs) into the standard treatment regimen for Philadelphia (Ph)-positive ALL significantly improved clinical outcomes. TKI-based induction chemotherapy, followed by allogeneic hematopoietic cell transplantation (HCT) during the first complete remission (CR), is the standard of care for ALL patients. However, treatment with TKIs alone or TKIs plus low-intensity chemotherapy can achieve CR in some patients. Although this strategy is not enough to induce a deeper molecular response, it can reduce the incidence of treatment-related mortality. Despite promising results from pediatric trials, allogeneic HCT remains an important component of the treatment strategy for Ph-positive adult ALL. However, improving the highly sensitive BCR-ABL1 assays and introducing immunotherapy may decrease the demand for allogeneic HCT. Nevertheless, the treatment of Ph-positive ALL is still challenging, especially in cases with relapsed and refractory disease. Potent TKIs and monoclonal antibodies, such as blinatumomab and inotuzumab, have improved patient outcomes in relapse and refractory cases of ALL. The introduction of effective agents, such as potent TKIs and monoclonal antibodies, may improve the possibility of remission in Ph-positive ALL patients and hopefully cure this disease.
\end{abstract}

Key Words Adult acute lymphoblastic leukemia, Philadelphia positive

\section{INTRODUCTION}

Acute lymphoblastic leukemia (ALL) is an aggressive hematological disease [1-4]. Combining high-dose chemotherapy and allogeneic hematopoietic cell transplantation (HCT) improved treatment outcomes in ALL patients [5-10]. Historically, Philadelphia chromosome (Ph)-positive cases of ALL, which comprise about $30 \%$ of all adult ALL cases, had a very poor prognosis. The incorporation of tyrosine kinase inhibitors (TKIs) in the treatment of ALL significantly improved treatment outcomes [6]. Nevertheless, the treatment of Ph-positive ALL is still challenging, especially in cases of relapsed and refractory disease. In this review, we will focus on the management of newly diagnosed Ph-positive ALL and highlight recent advances in the treatment of relapsed and refractory ALL.

\section{CURRENT STANDARD TREATMENT STRATEGIES FOR TRANSPLANT-ELIGIBLE PATIENTS}

TKI-based treatments improve the rates of complete remission (CR) in Ph-positive ALL patients. After the introduction of TKIs, an allogeneic HCT is performed in up to $80 \%$ of patients [11]. Despite the limitations of allogeneic HCT, such as severe graft-versus-host disease (GVHD) and other complications, it remains the standard of care for the treatment of Ph-positive ALL $[12,13]$. The UKALLXII/ECOG 2993 study revealed that adult Ph-positive ALL exhibited a significant response to allogeneic HCT [6, 14]. The GRAAPH-2005 trial compared the treatment outcomes after allogeneic HCT with those after autologous stem cell transplantation. The rates of five-year event-free survival and overall survival (OS) were $37 \%$ and $46 \%$, respectively. Furthermore, allogeneic HCT showed better survival outcomes in relapse-free survival (hazard ratio of 0.69 ) and OS (hazard ratio of 0.64) [15]. Other trials suggested a role 
for allogeneic HCT in the treatment of younger adult patients. $[6,16]$. All patients who were eligible for transplantation underwent allogeneic HCT after CR, and they reported high rates of survival.

Using RT-PCR to assess the minimal residual disease (MRD) in cases of BCR-ABL1 provides a useful method to identify high-risk patients. Early and deeper molecular responses before allogeneic HCT are associated with better clinical outcomes. A negative MRD score before allogeneic HCT was associated with a significantly improved survival rate and an incidence rate of $8 \%$ for disease relapse over a five-year period. In contrast, $39 \%$ of patients who did not undergo allogeneic HCT experienced relapse [17]. Kim et al. [8] reported that patients who achieved deeper molecular response during treatment with multiple chemotherapy drugs plus nilotinib before allogeneic HCT showed better long-term outcomes. The outcomes of MRD-positive patients at the time of allogeneic HCT were poorer than those of the MRD-negative cases [18-21].

\section{INITIATION OF TREATMENT; IS CHEMOTHERAPY NECESSARY?}

Although the incorporation of TKIs into chemotherapy is considered the standard treatment for cases of $\mathrm{Ph}$-positive ALL, the necessity of chemotherapy remains an important yet controversial issue, especially in older or fragile patients. The results of the early GIMEMA group study suggested that TKI monotherapy yielded a complete hematologic response (CHR) rate of $100 \%$ in $\mathrm{Ph}$-positive ALL patients [22]. Foá et al. [23] also reported a 100\% CHR rate after treatment with dasatinib (with steroid) monotherapy; however, $43 \%$ of patients relapsed after treatment. In the GIMEMA LAL 0904 study, with treatment schemes including imatinib plus steroids as induction and imatinib plus chemotherapy as consolidation therapy resulted in a CHR of 96\% [24]. All these studies showed relatively good toxicity profiles.

Several other studies reported combinations of TKIs with low-intensity chemotherapy. The outcomes of these trials were acceptable with CR rates between 95 and 100\% [5, $15,25]$. Chalandon et al. directly compared patient groups treated with TKIs plus low-intensity chemotherapy to TKIs plus conventional chemotherapy (GRAAPH-2005 trial). The rate of CHR was better in the group treated with low-intensity chemotherapy compared to standard chemotherapy (98.5\% vs. 91\%), and the low-intensity group had a decreased incidence of early toxic death $(1.7 \%$ vs. $6.7 \%, P=0.01)$. Although all patients in this study received high-dose methotrexate (MTX) and cytarabine at consolidation and the study protocol allowed allogeneic HCT after chemotherapy, long-term outcomes were also comparable between the two groups [15]. These studies suggested that low-intensity chemotherapy is as effective as intensive chemotherapy for $\mathrm{Ph}$-positive adult ALL patients. Furthermore, low-intensity chemotherapy led to reduced initial morbidity and mortality among patients.
Although TKI monotherapy and TKIs plus low-intensity chemotherapy have clinical benefits in $\mathrm{Ph}$-positive adult ALL patients, many patients relapsed after treatment. This finding may be due to the high incidence of TKI-resistant T315I mutations among patient populations [9, 23, 25]. TKIs with a higher potency, such as ponatinib, may be effective in treating patients that screen positive for these mutations. Unfortunately, only limited data regarding these findings are available [26]. Combining a TKI with immunotherapy can be a good choice for treatment. Recently, Chiaretti et al. [24] reported the results of a Phase II study focusing on treatment with dasatinib plus blinatumomab, a bispecific T-cell engager. In total, 63 patients were enrolled in the study; each patient received two to three cycles of blinatumomab treatment. Among these patients, the CR rate was $47 \%$ and the OS rate was $95 \%$ at 10 months.

\section{IS ALLOGENEIC HCT MANDATORY FOR LONG-TERM SURVIVAL?}

Allogeneic HCT is a long-established strategy for treating patients with $\mathrm{Ph}$-positive ALL. However, this treatment modality has numerous potential adverse effects such as GVHD, veno-occlusive disease, and infection. Younger patients without comorbidities can expect benefits from allogeneic HCT whereas older patients have an increased risk of treatment-related mortality. Although allogeneic HCT is generally considered to be immune to adverse genetic and cytogenetic mutations, some studies concluded that the results of allogeneic HCT in cases of T315I mutation and deletions of CKND2A/2B, PAX5 genes were poor despite allogeneic HCT [27-29].

The AALL0031 study reported the long-term outcomes of $\mathrm{Ph}$-positive pediatric ALL patients after allogeneic HCT. Survival outcomes were similar in patients with and without allogeneic HCT (five-year rates of disease-free survival were $70 \pm 12 \%$ in the without transplant group, $65 \pm 11 \%$ in the allogeneic HCT from related donor group, and $59 \pm 15 \%$ in the allogeneic HCT from unrelated donor group; $P=0.60$ ) [30]. Slayton et al. [31] reported positive outcomes for $\mathrm{Ph}$-positive pediatric ALL patients, with five-year OS rates of $86 \%$. In this study, the clinical outcomes of patients who underwent allogeneic HCT were comparable with those of patients who received chemotherapy alone.

Despite promising results in pediatric studies, the application of this strategy to $\mathrm{Ph}$-positive adult ALL patients is still being debated. In the UKALLXII/ECOG 2993 study, some patients who did not undergo transplantation sustained a long-term disease-free status after treatment. However, the majority of patients who underwent allogeneic HCT after CR achieved better outcomes (four-year OS rates of $50 \%$ in myeloablative conditioning transplant group compared with $39 \%$ in the reduced-intensity conditioning transplant group and $19 \%$ in the chemotherapy-only group) [6]. Ravandi et al. [16] conducted a U.S. intergroup multicenter trial focusing on 97 patients with $\mathrm{Ph}$-positive adult ALL. 
This study reported better survival rates in patients who underwent allogeneic HCT [relapse-free survival (RFS) and OS, $P=0.038$ and 0.037 , respectively].

Although limited data exists regarding the efficacy of the third-generation multi-TKI, ponatinib, Jabbour et al. [32] reported promising clinical results using Hyper-CVAD plus ponatinib treatment. Patients who underwent allogeneic HCT did not show a difference in OS whereas the three-year OS rate was $70 \%$ for the allogeneic HCT group versus $87 \%$ for the TKI maintenance group. Meanwhile, patients treated with deeper molecular responders had better long-term outcomes; the three-year OS rate for patients with a complete molecular response (CMR) was $81 \%$ after 3 months. This study introduced the possibility of a transplantation-free treatment strategy using a third-generation TKI. Furthermore, allogeneic HCT may benefit only MRD-positive patients; however, this question requires further investigation.

\section{NEW CHALLENGES IN RELAPSED PH-POSITIVE ALL TREATMENT}

Adding TKIs to chemotherapy regimens and following this with allogeneic HCT improved patient outcomes; unfortunately, the prognosis of relapsed and refractory cases of Ph-positive ALL is still very poor. Kadia et al. [33] reported a phase II study focusing on the use of MTX, vincristine, pegylated-asparaginase, and dexamethasone in relapsed and refractory ALL cases. This treatment regimen yielded a CR rate of $28 \%$ and an overall response rate of $39 \%$. The CR rate of newly diagnosed Ph-positive ALL patients is $>90 \%$ [13]; however, in cases of relapsed disease, we can expect a CR rate of 30 to $40 \%$ after the first round of salvage chemotherapy [34-36]. Survival outcomes among relapsed cases decrease after the first salvage treatment. Relapsed $\mathrm{Ph}$-positive ALL cases are often accompanied by mutations in the ABL1 kinase domain, such as the T315I mutation $[9,25]$. However, relapse may also occur without $A B L 1$ mutations. In addition to $A B L 1$ mutations, various signaling abnormalities may contribute to relapse.

The use of immunotherapy to treat relapsed and refractory cases of Ph-positive ALL has been less explored compared to the use of immunotherapy to treat cases of $\mathrm{Ph}$-negative ALL. In the Phase II ALCANTARA study, blinatumomab showed promising results in patients with $\mathrm{Ph}$-positive ALL and yielded a median OS of 7.1 months and RFS of 6.7 months. Sixteen out of the $45(36 \%)$ patients achieved CR (with 14 patients achieving CMR) regardless of prior TKI therapy. Meanwhile, 4 out of 10 patients (40\%) with a T315I mutation experienced CR. The authors concluded that blinatumomab showed effective antileukemic effects in relapsed and refractory $\mathrm{Ph}$-positive cases of ALL [37]. Assi et al. reported the results of a retrospective study on 13 patients with relapsed refractory Ph-positive ALL and chronic myeloid leukemia with reasonable clinical outcomes [38]. On comparing blinatumomab with the standard of care in the treatment of relapsed and refractory cases of $\mathrm{Ph}$-positive
ALL, the OS after blinatumomab treatment was superior to that associated with the standard of care (hazard ratio $=0.81$ ) [39].

Inotuzumab ozogamicin, a humanized anti-CD22 monoclonal antibody conjugated to calicheamicin, is another promising agent for treating ALL. In the Phase III randomized INO-VATE study, remission rates did not differ significantly between the inotuzumab ozogamicin group and the standard chemotherapy group among patients with $\mathrm{Ph}$-positive ALL [40]. Although this study failed to prove the clinical superiority of inotuzumab ozogamicin over standard chemotherapy regimens in patients with relapsed and refractory $\mathrm{Ph}$-positive ALL, inotuzumab ozogamicin showed favorable rates of $\mathrm{CR}$ (78.6\% vs. $44.4 \%, P=0.08$ ). The introduction of powerful drugs, such as blinatumomab and inotuzumab, and innovative prospective clinical trials, such as TKIs with immunotherapy or TKIs with other combinations of targeted therapy, and the adoption of frontline immunotherapy is currently ongoing.

\section{FUTURE DIRECTIONS}

The incorporation of TKIs in the treatment of Ph-positive ALL significantly improved clinical outcomes. However, the clinical outcomes in patients with relapsed and refractory $\mathrm{Ph}$-positive cases of ALL are still grave. The rapid development of monoclonal antibodies led to a breakthrough in the treatment of relapsed and refractory cases of ALL. Future studies should reveal the optimal combinations of monoclonal antibodies with or without standard chemotherapy. Currently, studies using monoclonal antibodies to treat newly diagnosed cases of $\mathrm{Ph}$-positive ALL are ongoing. If these potent monoclonal antibodies can reduce the intensity of standard chemotherapy regimens, treatment-related mortality rates may reduce during treatment. Moreover, if these approaches can achieve deeper and durable molecular responses, it may decrease the demand for allogeneic HCT.

There are several issues regarding chimeric antigen receptor (CAR) T-cell therapies for Ph-positive ALL. Currently, the anti-CD19 CAR T-cell is approved for the treatment of relapsed and refractory ALL cases in children and young adults. Long-term follow-up data on CD19 CAR T-cell therapy for treating relapsed ALL cases showed impressive outcomes, especially in $16 \mathrm{Ph}$-positive adult ALL patients [41]. The positive effects of CAR T-cell treatments are undeniable, but there is debate over whether CAR T-cell therapy can replace allogeneic HCT [42]. Whether CAR T-cell therapy is to be applied before or after immunotherapy is another question because there are concerns about target-antigen modulation after immunotherapy [43].

\section{CONCLUSION}

Treatment strategies for $\mathrm{Ph}$-positive ALL patients are rapidly changing. Fortunately, the introduction of effective 
agents, such as potent TKIs and monoclonal antibodies, may improve the possibility of remission in Ph-positive ALL patients and hopefully cure this disease.

\section{Authors' Disclosures of Potential Conflicts of Interest}

No potential conflicts of interest relevant to this article were reported.

\section{REFERENCES}

1. Annino L, Vegna ML, Camera A, et al. Treatment of adult acute lymphoblastic leukemia (ALL): long-term follow-up of the GIMEMA ALL 0288 randomized study. Blood 2002;99:863-71.

2. Kantarjian H, Thomas D, O'Brien S, et al. Long-term follow-up results of hyperfractionated cyclophosphamide, vincristine, doxorubicin, and dexamethasone (Hyper-CVAD), a dose-intensive regimen, in adult acute lymphocytic leukemia. Cancer 2004; 101:2788-801.

3. Thomas X, Boiron JM, Huguet F, et al. Outcome of treatment in adults with acute lymphoblastic leukemia: analysis of the LALA-94 trial. J Clin Oncol 2004;22:4075-86.

4. Rowe JM, Buck G, Burnett AK, et al. Induction therapy for adults with acute lymphoblastic leukemia: results of more than 1500 patients from the international ALL trial: MRC UKALL XII/ECOG E2993. Blood 2005;106:3760-7.

5. Bassan R, Rossi G, Pogliani EM, et al. Chemotherapy-phased imatinib pulses improve long-term outcome of adult patients with Philadelphia chromosome-positive acute lymphoblastic leukemia: Northern Italy Leukemia Group protocol 09/00. J Clin Oncol 2010;28:3644-52.

6. Fielding AK, Rowe JM, Buck G, et al. UKALLXII/ECOG2993: addition of imatinib to a standard treatment regimen enhances long-term outcomes in Philadelphia positive acute lymphoblastic leukemia. Blood 2014;123:843-50.

7. Daver N, Thomas D, Ravandi F, et al. Final report of a phase II study of imatinib mesylate with hyper-CVAD for the front-line treatment of adult patients with Philadelphia chromosomepositive acute lymphoblastic leukemia. Haematologica 2015; 100:653-61.

8. Kim DY, Joo YD, Lim SN, et al. Nilotinib combined with multiagent chemotherapy for newly diagnosed Philadelphiapositive acute lymphoblastic leukemia. Blood 2015;126:746-56.

9. Ravandi F, O'Brien SM, Cortes JE, et al. Long-term follow-up of a phase 2 study of chemotherapy plus dasatinib for the initial treatment of patients with Philadelphia chromosome-positive acute lymphoblastic leukemia. Cancer 2015;121:4158-64.

10. Wang J, Jiang Q Xu LP, et al. Allogeneic stem cell transplantation versus tyrosine kinase inhibitors combined with chemotherapy in patients with Philadelphia chromosome-positive acute lymphoblastic leukemia. Biol Blood Marrow Transplant 2018; 24:741-50.

11. Litzow MR, Fielding AK, Luger SM, et al. The evolving role of chemotherapy and hematopoietic cell transplants in $\mathrm{Ph}$-positive acute lymphoblastic leukemia in adults. Bone Marrow Transplant
2017;52:1592-8.

12. Stock W. Current treatment options for adult patients with Philadelphia chromosome-positive acute lymphoblastic leukemia. Leuk Lymphoma 2010;51:188-98.

13. Fielding AK. Current treatment of Philadelphia chromosome-positive acute lymphoblastic leukemia. Hematology Am Soc Hematol Educ Program 2011;2011:231-7.

14. Fielding AK, Rowe JM, Richards SM, et al. Prospective outcome data on 267 unselected adult patients with Philadelphia chromosome-positive acute lymphoblastic leukemia confirms superiority of allogeneic transplantation over chemotherapy in the pre-imatinib era: results from the International ALL Trial MRC UKALLXII/ECOG2993. Blood 2009;113:4489-96.

15. Chalandon $Y$, Thomas X, Hayette S, et al. Randomized study of reduced-intensity chemotherapy combined with imatinib in adults with Ph-positive acute lymphoblastic leukemia. Blood 2015;125:3711-9.

16. Ravandi F, Othus M, O'Brien SM, et al. US Intergroup study of chemotherapy plus dasatinib and allogeneic stem cell transplant in Philadelphia chromosome positive ALL. Blood Adv 2016;1: 250-9.

17. Lussana F, Intermesoli T, Gianni F, et al. Achieving molecular remission before allogeneic stem cell transplantation in adult patients with Philadelphia chromosome-positive acute lymphoblastic leukemia: impact on relapse and long-term outcome. Biol Blood Marrow Transplant 2016;22:1983-7.

18. Lee S, Kim DW, Cho BS, et al. Impact of minimal residual disease kinetics during imatinib-based treatment on transplantation outcome in Philadelphia chromosome-positive acute lymphoblastic leukemia. Leukemia 2012;26:2367-74.

19. Nicolini FE, Basak GW, Kim DW, et al. Overall survival with ponatinib versus allogeneic stem cell transplantation in Philadelphia chromosome-positive leukemias with the T315I mutation. Cancer 2017;123:2875-80.

20. Nishiwaki S, Imai K, Mizuta S, et al. Impact of MRD and TKI on allogeneic hematopoietic cell transplantation for $\mathrm{Ph}+\mathrm{ALL}$ : a study from the adult ALL WG of the JSHCT. Bone Marrow Transplant 2016;51:43-50.

21. Yoon JH, Min GJ, Park SS, et al. Minimal residual disease-based long-term efficacy of reduced-intensity conditioning versus myeloablative conditioning for adult Philadelphia-positive acute lymphoblastic leukemia. Cancer 2019;125:873-83.

22. Vignetti M, Fazi P, Cimino G, et al. Imatinib plus steroids induces complete remissions and prolonged survival in elderly Philadelphia chromosome-positive patients with acute lymphoblastic leukemia without additional chemotherapy: results of the Gruppo Italiano Malattie Ematologiche dell'Adulto (GIMEMA) LAL0201-B protocol. Blood 2007;109:3676-8.

23. Foà R, Vitale A, Vignetti M, et al. Dasatinib as first-line treatment for adult patients with Philadelphia chromosome-positive acute lymphoblastic leukemia. Blood 2011;118:6521-8.

24. Chiaretti S, Vitale A, Vignetti M, et al. A sequential approach with imatinib, chemotherapy and transplant for adult $\mathrm{Ph}+$ acute lymphoblastic leukemia: final results of the GIMEMA LAL 0904 study. Haematologica 2016;101:1544-52.

25. Rousselot P, Coudé MM, Gokbuget N, et al. Dasatinib and low-intensity chemotherapy in elderly patients with Philadelphia 
chromosome-positive ALL. Blood 2016;128:774-82.

26. Jabbour E, Kantarjian H, Ravandi F, et al. Combination of hyper-CVAD with ponatinib as first-line therapy for patients with Philadelphia chromosome-positive acute lymphoblastic leukaemia: a single-centre, phase 2 study. Lancet Oncol 2015; 16:1547-55.

27. Martinelli G, Iacobucci I, Storlazzi CT, et al. IKZF1 (Ikaros) deletions in BCR-ABL1-positive acute lymphoblastic leukemia are associated with short disease-free survival and high rate of cumulative incidence of relapse: a GIMEMA AL WP report. J Clin Oncol 2009;27:5202-7.

28. Pfeifer H, Raum K, Markovic S, et al. Genomic CDKN2A/2B deletions in adult $\mathrm{Ph}+\mathrm{ALL}$ are adverse despite allogeneic stem cell transplantation. Blood 2018;131:1464-75.

29. Fedullo AL, Messina M, Elia L, et al. Prognostic implications of additional genomic lesions in adult Philadelphia chromosomepositive acute lymphoblastic leukemia. Haematologica 2019;104: 312-8.

30. Schultz KR, Carroll A, Heerema NA, et al. Long-term follow-up of imatinib in pediatric Philadelphia chromosome-positive acute lymphoblastic leukemia: Children's Oncology Group study AALL0031. Leukemia 2014;28:1467-71.

31. Slayton WB, Schultz KR, Kairalla JA, et al. Dasatinib plus intensive chemotherapy in children, adolescents, and young adults with philadelphia chromosome-positive acute lymphoblastic leukemia: results of Children's Oncology Group Trial AALL0622. J Clin Oncol 2018;36:2306-14.

32. Jabbour E, Short NJ, Ravandi F, et al. Combination of hyper-CVAD with ponatinib as first-line therapy for patients with Philadelphia chromosome-positive acute lymphoblastic leukaemia: long-term follow-up of a single-centre, phase 2 study. Lancet Haematol 2018;5:e618-27.

33. Kadia TM, Kantarjian HM, Thomas DA, et al. Phase II study of methotrexate, vincristine, pegylated-asparaginase, and dexamethasone $(\mathrm{MOpAD})$ in patients with relapsed/refractory acute lymphoblastic leukemia. Am J Hematol 2015;90:120-4.

34. Thomas DA, Kantarjian H, Smith TL, et al. Primary refractory and relapsed adult acute lymphoblastic leukemia: characteristics, treatment results, and prognosis with salvage therapy. Cancer 1999;86:1216-30.

35. Fielding AK, Richards SM, Chopra R, et al. Outcome of 609 adults after relapse of acute lymphoblastic leukemia (ALL); an MRC UKALL12/ECOG 2993 study. Blood 2007;109:944-50.

36. Tavernier E, Boiron JM, Huguet F, et al. Outcome of treatment after first relapse in adults with acute lymphoblastic leukemia initially treated by the LALA-94 trial. Leukemia 2007;21:1907-14.

37. Martinelli G, Boissel N, Chevallier P, et al. Complete hematologic and molecular response in adult patients with relapsed/refractory philadelphia chromosome-positive B-precursor acute lymphoblastic leukemia following treatment with blinatumomab: results from a phase II, single-arm, multicenter study. J Clin Oncol 2017; 35:1795-802.

38. Assi R, Kantarjian H, Short NJ, et al. Safety and efficacy of blinatumomab in combination with a tyrosine kinase inhibitor for the treatment of relapsed Philadelphia chromosome-positive leukemia. Clin Lymphoma Myeloma Leuk 2017;17:897-901.

39. Rambaldi A, Ribera JM, Kantarjian HM, et al. Blinatumomab compared with standard of care for the treatment of adult patients with relapsed/refractory Philadelphia chromosome-positive B-precursor acute lymphoblastic leukemia. Cancer 2020;126: 304-10.

40. Kantarjian HM, DeAngelo DJ, Stelljes M, et al. Inotuzumab ozogamicin versus standard therapy for acute lymphoblastic leukemia. N Engl J Med 2016;375:740-53.

41. Park JH, Rivière I, Gonen M, et al. Long-term follow-up of CD19 CAR therapy in acute lymphoblastic leukemia. N Engl J Med 2018;378:449-59.

42. Jacoby $\mathrm{E}$. The role of allogeneic HSCT after CAR T cells for acute lymphoblastic leukemia. Bone Marrow Transplant 2019;54(Suppl 2):810-4.

43. Ruella M, Maus MV. Catch me if you can: leukemia escape after CD19-directed T cell immunotherapies. Comput Struct Biotechnol J 2016;14:357-62. 